\title{
Morbidity and Mortality of Very Low Birth Weight Babies in a Tertiary Level NICU
}

\author{
Kaur A ${ }^{1}$, Thapar $K^{2}$, Chhabra GS ${ }^{3}$, Jaslean ${ }^{4}$
}

\begin{abstract}
Introduction: The WHO defines Very low birth weight babies as birth weight $<1500$ grams at birth irrespective of gestational age. The primary cause of VLBW is premature birth.The commonest conditions in this group include aspiration, infections, Hyaline membrane disease. Early onset sepsis is an important cause of illness and death among infants with very low birth weights. The objectives were to study the morbidity and mortality of very low birth weight babies in teritary level NICU in Amritsar and the sociodemographic, obstetric and neonatal variables affecting survival of VLBW infants. Materials and Method: It was an observational study and conducted in NICU of SGRDIMS\&R, Amritsar, over the period of 18 months ( $1^{\text {st }}$ October 2013 to $31^{\text {st }}$ March 2015). A total of 75 VLBW neonates were observed during this period and neonatal outcome was assessed. Data so obtained was statistically analysed by Microsoft SPSS, Version 17.0.Chi Square test was applied to the data. Results: Preterm VLBW babies had high incidence of morbidities. Sepsis was the major cause (77.3\%) of morbidity in VLBW neonates followed by HMD (66.7\%) and NNJ (65.3\%) of the neonate. Sepsis along with other factors like IVH, Pneumothorax, NEC play an important role in neonatal mortality. Conclusion: The most common cause of VLBW babies was prematurity. The mortality rate among VLBW neonates was $12 \%$. Sepsis, HMD metabolic complications like hypoglycaemia and hypocalcemia were the major contributing factors towards mortality.
\end{abstract}

Key words: Preterm babies, Sepsis, Very low birth weight, Morbidity, Mortality.

\section{Introduction}

$\mathrm{N}$ eonatal period is the most vulnerable period of life due to different diseases, which in most cases are preventable ${ }^{1}$.The WHO defines Very low birth weight babies (VLBW) as birth weight $<1500$ grams at birth irrespective of gestational age. The incidence of VLBW babies is less than $2 \%$ of the births globally ${ }^{2}$. In India, VLBW babies constitute $4 \%$ to $7 \%$ of the live births and approximately $30 \%$ of neonatal death ${ }^{3}$. The primary cause of VLBW is premature birth. Other factors that can contribute to the risk of VLBW include: Age, Multiple birth babies, Maternal health, Mothers of lower socioeconomic status.
${ }^{1}$ Dr. Amandeep Kaur, MBBS, MD, ${ }^{2} \mathrm{Dr}$ Karuna Thapar, MBBS, MD. Professor and Head, ${ }^{3} \mathrm{Dr}$. Gurpreet Singh Chhabra, MBBS, MD, Associate Professor, ${ }^{4} \mathrm{Dr}$. Jaslean, MBBS, MD, Senior Resident. All from the Department of Paediatrics, Sri Guru Ram Das Institute of Medical Science and Research, Amritsar, Punjab, India.

Address for correspondence:

Dr. Amandeep Kaur

Sri Guru Ram Das Institute Of Medical Science and Research

Amritsar Punjab

PIN 14300, India

Tel: +918872411863

E-mail: amandeepk441@gmail.com

\section{How to cite}

Kaur A, Thapar K, Chhabra GS, Jaslean. Morbidity and Mortality of Very Low Birth Weight Babies in a Tertiary Level NICU. J Nepal Paediatr Soc 2015;35(3):257-263.

doi: http://dx.doi.org/10.3126/jnps.v35i3.13984

This work is licensed under a Creative Commons Attribution 3.0 License.

(c) (i)

VLBW babies are mostly premature, requiring extra care to prevent heat loss because of thin skin and an increased surface area to body-weight ratio. In VLBW babies, respiratory problems are very common. The commonest conditions in this group include aspiration, infections, Hyaline membrane disease (HMD). VLBW may present with hypotension due to hypovolemia, vasodilatation due to sepsis. Patent Ductus Arteriosus (PDA) is common and may lead to congestive heart failure ${ }^{5}$. VLBW presented with slow rates of weight gain due to gut immaturity with decreased motility, enzyme deficiencies and increase risk of necrotizing enterocolitis. VLBW 
babies are vulnerable to infections because of epidermal barrier immaturity, poor defense mechanisms. Early onset sepsis is an important cause of illness and death among infants with very low birth weights (less than $1500 \mathrm{~g})^{6}$. Seizures occur in 20 to $50 \%$ of preterm infants and these are the clinical manifestation of serious underlying neurological disease and can cause additional brain injury.

The Objectives of this study were to study the morbidity and mortality of very low birth weight babies and to study the sociodemographic, obstetric and neonatal variables affecting survival of VLBW infants in teritary a level NICU in Sri Guru Ram Das Institute of Medical Science and Research (SGRDIMSR )Amritsar.

\section{Material and Methods}

All VLBW neonates with birth weight between 1001 to 1500 gms admitted in NICU, SGRDIMSR with no major congenital anomalies were included in this study. Their mode and place of delivery was recorded. Neonatal weight was done by electronic weighing scale. Gestational age was assessed by Ballard scoring and the neonates were divided into: Term and Preterm.

Maternal factors affecting neonatal outcome were assessed as follows

1. Mothers were divided into three categories according to the age as $<20$ years, $20-30$ years, $>30$ years, since age $<20$ years and $>30$ years are known to adversely affect neonatal outcome.

2. Maternal height was measured using stadiometer without shoes and categorised as $>150 \mathrm{~cm}$ and $<150 \mathrm{~cm}$ according to WHO criteria.

3. Maternal antenatal complications which are known to affect neonatal outcome were assessed Socio-economic status was derived from the Modified-Kuppuswamy scale.

Neonatal morbidity factors were assessed and are as follows:

1. APGAR score at 1 and 5 minutes was observed.

2. PNA was assessed as APGAR score of less than 5 at $1 \mathrm{~min}$ of age.

3. Respiratory Complications:-

a. Respiratory distress was assessed by Downe's score in term neonates and Silverman-
Anderson score in case of Preterm neonates. HMD was diagnosed by skiagram of chest showing symmetrical reticulogranular pattern, ground glass opacity or complete white out.

b. Pneumothorax was diagnosed by respiratory distress, Chest X-Ray.

4. Metabolic Complications:-

a. Hypoglycemia was diagnosed by blood glucose level $<40 \mathrm{mg} / \mathrm{dl}$, done by standard glucometer by heel prick method. Hypocalcemia was defined as serum calcium $<7 \mathrm{mg} / \mathrm{dl}$.

b. Hyponateremia was defined as serum sodium $<120 \mathrm{mEq} / \mathrm{l}$

c. Hypocalcemia was defined as serum calcium < $7 \mathrm{mg} / \mathrm{dl}$.

5. CVS Complication-

a. PDA was diagnosed clinically on the basis of clinical examination (loud systolic murmur, widened pulse pressures, a decreased systemic blood pressure, bounding pulses, a hyperactive precordium, and an increased respiratory effort).

6. CNS Complication:- IVH was diagnosed on crainal ultrasonography.

7. GIT Complication:- NEC was diagnosed clinically on basis of Modified Bell's criteria.

8. Haematological Complications:- Anaemia: $\mathrm{Hb}$ or PCV value below expected range for the gestational and chronological age. NNJ:- Any level of serum bilirubin that require intervention.

9. Septicaemia was defined on the basis of clinical features and positive findings of septic screen (PBF-toxic granules, band forms, shift to left, raised CRP or bacteriological positive blood or CSF culture.

Data so obtained was statistically analysed by Microsoft SPSS, Version 17.0.Chi Square test was applied to the data. Permission was taken from institute review. Consent was taken from patient's parent.

\section{Results}

Seventy five VLBW neonates were observed during a period of 18 months and the following inferences were drawn:- 
Table 1: Correlation of morbidity factors and sex predilection.

\begin{tabular}{|c|c|c|c|c|}
\hline Morbidity Factors & No of Cases & Male (N=55) & Female (N=20) & $p$-value \\
\hline Sepsis & 58 & $42(56 \%)$ & $16(21.3 \%)$ & 0.739 \\
\hline NNJ & 49 & $32(42.6 \%)$ & $17(22.7 \%)$ & 0.031 \\
\hline PNA & 12 & $8(10.6 \%)$ & $4(5.3 \%)$ & 0.569 \\
\hline HMD & 50 & $34(45.3 \%)$ & $16(21.3 \%)$ & 0.140 \\
\hline Apnea & 15 & $11(14.6 \%)$ & $4(5.3 \%)$ & 1.000 \\
\hline Pneumothorax & 17 & $16(21.3 \%)$ & $1(1.3 \%)$ & 0.028 \\
\hline IVH & 4 & $4(5.3 \%)$ & 0 & 0.302 \\
\hline HIE & 7 & $4(5.3 \%)$ & $3(4 \%)$ & 0.189 \\
\hline NEC & 15 & $13(17.3 \%)$ & $2(2.6 \%)$ & 0.192 \\
\hline PDA & 5 & $3(4 \%)$ & $2(2.6 \%)$ & 0.485 \\
\hline Hypoglycemia & 44 & $42(56 \%)$ & $16(21.3 \%)$ & 0.739 \\
\hline Hypocalcemia & 37 & $28(37.3 \%)$ & $9(24.3 \%)$ & 0.651 \\
\hline
\end{tabular}

* NNJ: Neonatal Jaumdice, PNA: Perinatal Asphyxia, HMD: Hyaline Membrane Disease, IVH: Intra Ventricular Haemorrhage, HIE: Hypoxic Ischaemic Encephalopathy, NEC: Necrotising Enterocolitis, PDA: Patent Ductus Arteriosus,

Table 2: Correlation of morbidity factors with gestational age.

\begin{tabular}{|c|c|c|c|c|}
\hline Morbidity Factors & No of Cases & Term (N=5) & Preterm (N=70) & Total \\
\hline Sepsis & 58 & $4(5.3 \%)$ & $54(72 \%)$ & 0.883 \\
\hline NNJ & 49 & 0 & $49(65.3 \%)$ & 0.001 \\
\hline PNA & 12 & 0 & $12(16 \%)$ & 0.312 \\
\hline HMD & 50 & $3(4 \%)$ & $47(62.6 \%)$ & 0.743 \\
\hline Apnea & 15 & 0 & $15(20 \%)$ & 0.247 \\
\hline Pneumothorax & 17 & $2(11.8 \%)$ & $15(20 \%)$ & 0.338 \\
\hline IVH & 4 & $1(1.3 \%)$ & $3(4 \%)$ & 0.200 \\
\hline HIE & 7 & $1(1.3 \%)$ & $6(8 \%)$ & 0.809 \\
\hline NEC & 15 & $3(4 \%)$ & $12(16 \%)$ & 0.021 \\
\hline PDA & 5 & 0 & 5 & 0.536 \\
\hline Hypoglycemia & 44 & $3(4 \%)$ & $41(54.6 \%)$ & 0.950 \\
\hline Hypocalcemia & 37 & $3(4 \%)$ & $34(45.3 \%)$ & 0.621 \\
\hline
\end{tabular}

Table 3: Correlation of morbidities with antenatal complications in the mothers of VLBW babies

\begin{tabular}{|c|c|c|c|}
\hline Morbidity Factors & Complications & No Complications & $p$-value \\
\hline VLBW babies & 46 & 29 & 0.05 \\
\hline Sepsis & $39(52 \%)$ & $19(25.3 \%)$ & 0.600 \\
\hline NNJ & $29(38.6 \%)$ & $20(26.6 \%)$ & 0.019 \\
\hline PNA & $11(14.6 \%)$ & $1(1.3 \%)$ & 0.502 \\
\hline HMD & $3(4 \%)$ & $47(62.6 \%)$ & 0.635 \\
\hline Apnea & $10(13.3 \%)$ & $5(6.67 \%)$ & 0.169 \\
\hline Pneumothorax & $8(10.7 \%)$ & $9(12.0 \%)$ & 0.831 \\
\hline IVH & $3(4 \%)$ & $1(1.3 \%)$ & 0.603 \\
\hline HIE & $4(5.3 \%)$ & $3(4 \%)$ & 0.906 \\
\hline NEC & $9(12.0 \%)$ & $6(8.0 \%)$ & 0.949 \\
\hline PDA & $3(4 \%)$ & $2(2.6 \%)$ & 0.635 \\
\hline Hypoglycemia & $26(34.6 \%)$ & $18(24 \%)$ & 0.080 \\
\hline Hypocalcemia & $19(25.3 \%)$ & $18(24 \%)$ & \\
\hline
\end{tabular}


Table 4: Correlation of morbidities with 1 min APGAR score of VLBW babies.

\begin{tabular}{|c|c|c|c|}
\hline Morbidity Factors & 1 Min Apgar Score(<5) & $\mathbf{1}$ Min Apgar Score(>5) & $\boldsymbol{p}$-value \\
\hline VLBW babies & 7 & 68 & 0.695 \\
\hline Sepsis & $5(6.67 \%)$ & $53(70.6 \%)$ & 0.722 \\
\hline NNJ & $5(6.67 \%)$ & $44(58.7 \%)$ & $<0.001$ \\
\hline PNA & $6(8 \%)$ & $6(8 \%)$ & 0.133 \\
\hline HMD & $6(8 \%)$ & $44(58.7 \%)$ & 0.165 \\
\hline Apnea & 0 & $15(20 \%)$ & 0.133 \\
\hline Pneumothorax & 0 & $17(22.6 \%)$ & $<0.001$ \\
\hline IVH & $1(1.3 \%)$ & $3(4 \%)$ & $<0.001$ \\
\hline HIE & $6(8 \%)$ & $1(1.3 \%)$ & 0.165 \\
\hline
\end{tabular}

Table 5: Correlation of morbidities with 5 min APGAR score of VLBW babies.

\begin{tabular}{|c|c|c|c|}
\hline Morbidity Factors & 5 Min Apgar Score (<5) & 5 Min Apgar Score (>5) & Total \\
\hline VLBW babies & 6 & 69 & 0.515 \\
\hline Sepsis & $4(6.9 \%)$ & $54(72 \%)$ & 0.334 \\
\hline NNJ & $5(6.67 \%)$ & $44(58.7 \%)$ & $<0.001$ \\
\hline PNA & $6(8 \%)$ & $6(8 \%)$ & 0.069 \\
\hline HMD & 0 & $49(65.3 \%)$ & 0.202 \\
\hline Apnea & $3(4 \%)$ & $15(20 \%)$ & 0.167 \\
\hline Pneumothorax & 0 & $17(22.6 \%)$ & $<0.001$ \\
\hline IVH & $1(1.3 \%)$ & $3(0.04 \%)$ & $<0.001$ \\
\hline HIE & $5(6.67 \%)$ & $2(2.6 \%)$ & 0.202 \\
\hline NEC & 0 & $15(20 \%)$ & \\
\hline
\end{tabular}

Sepsis was the major cause $(77.3 \%)$ of morbidity in VLBW neonates followed by HMD (66.7\%) and NNJ (65.3\%) of the neonates. In neurological disease HIE, PNA and IVH contributed to $6.7 \%, 9.3 \%$ and $5.3 \%$ of the cases respectively. PDA, NEC and metabolic complications like hypoglycemia, hypocalcemia were seen in $6.7 \%, 20 \%, 58.7 \%$ and $49.3 \%$ of the babies respectively. Many factors contribute towards mortality in VLBW babies. Sepsis along with other factors play an important role in neonatal mortality $(p=0.083)$. Out of the total nine mortalities, three cases were associated with IVH ( $p$-value of $<0.001)$. Pneumothorax was an associated factor in three cases ( $p$-value $<0.001$, statistically significant). PNA and NNJ were contributing factors in four cases each with $p$-value of 0.132 and 0.160 respectively.

Results showed that $73.3 \%$ of neonates were male and $26.7 \%$ were female. All morbidity factors were seen more in male neonates as compared to female. PNA was seen in $10.6 \%$, NNJ in $42.6 \%$, HMD in $45.3 \%$, apnea in $14.6 \%$, HIE in $5.3 \%$ of males as compared to females with PNA in 5.3\%, NNJ in 22.7\%, HMD in 21.3\%, $\mathrm{HIE}$ in $4 \%$ respectively but none of the association was statistically significant. Seven males out of 55 died and 2 out of 20 females died ( $p=0.748$, not significant).
Similarly $93.3 \%$ of VLBW in our study were preterm. Metabolic complications like hypocalcemia and hypoglycemia were seen in preterm VLBW babies with incidence of $54.6 \%$ and $45.3 \%$ respectively. NEC and NNJ were statistically significantly seen in preterm neonates ( $p=0.021$ and $p<0.001$, respectively). Out of 5 term born neonates, one expired and eight out of 70 preterm neonates expired. $(p=0.569)$.

Out of all, $9.3 \%$ of the total neonates had $<5$ APGAR score at $1 \mathrm{~min}$ and $8 \%$ had $<5$ APGAR score at $5 \mathrm{~min}$. Out of total VLBW babies, six had perinatal asphyxia with low APGAR score at 1 min with $p$ value of $<0.001$ (statistically significant). CNS morbidities like HIE and IVH with low APGAR score at $1 \mathrm{~min}$ were found in $8 \%$ and $1.3 \%$ of total cases with $p$ value $<0.001$ (statistically very significant). In all VLBW babies, PNA, IVH and HIE with low APGAR score at 5 minute were seen in $8 \%, 6.7 \%$ and $1.3 \%$ respectively with $p$ value $<0.001$ (statistically significant).1 min APGAR score and 5 min Apgar score of $<5$ had $22.2 \%$ and $11.1 \%$ contribution to the neonatal mortality respectively with respective $p$-values of 0.156 and 0.714 .

Out of all the mothers $93.3 \%$ were in $20-30$ yrs age group, $5.3 \%$ were $<20 y$ rs and only $1.3 \%$ of mothers 
were $>30$ yrs old. Majority of VLBW babies (70) were born to the mothers with 20-30 yrs of age group. Hence maximum morbities and mortalities were seen in neonates of mothers who belonged to this age group.

Antenatal complications like fever, drug intake, oligohydraminos, PROM, anaemia, were seen in mothers of 2 (2.7\%), 1 (1.3\%), 46 (61.3\%), 10 (13.3\%), 16 (21.3\%) VLBW neonates, respectively. Oligohydraminos was the major maternal factor contributing to very low birth weight neonates. Sepsis (52\%) and PNA (14.6\%) were seen in neonates of mothers with antenatal complications ( $p$-value 0.05 and 0.01 , respectively). $36 \%$ of the mothers were from upper middle class strata and $58.7 \%$ from upper lower strata. Six out of nine cases who died belong to Upper lower class families $(p=0.713)$.

Out of all babies, 94.7\% were hospital delivered and $5.3 \%$ home delivered. VLBW babies are high risk babies. Most of the babies were hospital born. So we could not compare morbidities with place of delivery. $56 \%$ of the VLBW babies were born by LSCS while $44 \%$ were born by NVD. Sepsis and NNJ were found in $49.3 \%$ and $25.3 \%$ of the total cases born by LSCS as compared to $28 \%$ and $40 \%$ of the total cases born by NVD with $p$ value of 0.01 and 0.211 respectively. Perinatal asphyxia and pneumothorax were seen in $16 \%$ and $18.6 \%$ of total cases born by LSCS ( $p$ value of $<0.001$, statistically significant). NEC, PDA and hypoglycemia were seen in $14.6 \%, 4 \%$ and $32 \%$ of LSCS born VLBW babies and in $5.3 \%, 2.6 \%, 26.7 \%$ of NVD born VLBW babies with $p$ value of $0.011,0.015,0.028$ respectively. (statistically significant). 5 out of 9 deaths were found in the babies born by LSCS and 4 out of 9 deaths were seen in the babies born by NVD ( $p=0.977)$.

Table 6: Outcome of study cases during the study period.

\begin{tabular}{|c|c|c|}
\hline Neonatal outcome & No of Cases & Percentage (\%) \\
\hline Satisfactory & 56 & $74.7 \%$ \\
\hline Left in between & 10 & $13.3 \%$ \\
\hline Death & 9 & $12 \%$ \\
\hline
\end{tabular}

Amongst all cases, $74.7 \%$ of the cases were discharged in satisfactory condition while $12 \%$ died during this study and $13.3 \%$ left the study in between.

\section{Discussion}

All in all, $73.3 \%$ of neonates were males and $26.7 \%$ were females. All morbid factors were seen more in male neonates as compared to female. In this study, 9.3\% of male neonates and $2.6 \%$ of females died. Brothwood et al confirmed the "relative vulnerability of boys to perinatal mortality and morbidity" They observed a higher rate of mortality and postnatal complications in very low birthweight boys than in girls ${ }^{9}$.

The primary cause of VLBW is premature birth. In this study, $93.3 \%$ of neonates were preterm. We observed that the preterm VLBW neonates had more frequent incidence of morbidities like sepsis (72\%), HMD (62.6\%), PNA (16\%), and apnea (20\%). NNJ (65.3\%) and $\mathrm{NEC}(16 \%)$ were seen in preterm neonates with $\mathrm{p}$ value $<0.05$ (statistically significant). Khan et al also found that preterm babies had more morbidities like metabolic derangement, neonatal jaundice, respiratory distress syndrome, intraventricular haemorrhage ${ }^{10}$. Mortality was seen in $10.6 \%$ of preterm as compared to $1.3 \%$ of term neonates (statistically not significant). Mannan et al found in their study that preterm VLBW babies and their mortality rate were higher than term babies, similar to our study ${ }^{8}$.

VLBW neonates born to the mothers with antenatal complications. It was observed that $23.9 \%$ of neonates born to mothers with antenatal complications had perinatal asphyxia with $p$-value of 0.019 (statistically significant). Sepsis was seen in $52 \%$ neonates born to mothers with antenatal complications ( $p=.05)$. All these morbidities can be decreased if there are adequate antenatal checkups, proper management of antenatal complications. Mannan et al concluded the similar result that the the mortality rate was higher in the babies who had antenatal maternal problem than no maternal problems babies ${ }^{8}$.

VLBW babies are high risk babies. Most of the babies were hospital born in our study. So we could not compare morbidities with place of delivery. In our study sepsis, neonatal jaundice, perinatal asphyxia, HMD, apnea, IVH, HIE, NEC were seen in 76\%, 60\%, $16 \%, 65.3 \%, 16 \%, 6.9 \%, 9.3 \%$ and $20 \%$ of total cases in hospital born neonates respectively. Mannan et al found that the maximum VLBW babies who died during hospital stay had multiple problems and mortality was varied $^{8}$.

Sepsis and NNJ were found in $49.3 \%$ and $25.3 \%$ of the total cases born by LSCS as compared to $28 \%$ and $40 \%$ of the total cases born by NVD with $p$ value of 0.01 and 0.211 respectively. Perinatal asphyxia and pneumothorax were seen in $16 \%$ and $18.6 \%$ of total cases born by LSCS with $p$ value of $<0.001$ that was statistically significant. NEC, PDA and hypoglycemia were seen in $14.6 \%, 4 \%$ and $32 \%$ of LSCS born VLBW babies and in $5.3 \%, 2.6 \%, 26.7 \%$ of NVD born VLBW babies with $p$-value of $0.011,0.015,0.028$ respectively. 
(statistically significant). MH Malloy found that the incidence of hyaline membrane disease among VLBW babies delivered by primary cesarean section compared with those delivered vaginally ${ }^{11}$.

In VLBW neonates, PNA was found in $8 \%$ cases with low APGAR score at 1 and 5 minute $(p<.001)$. CNS morbidities like IVH and HIE were significantly found in VLBW babies with low APGAR score at $1 \mathrm{~min}$ and $5 \mathrm{~min}$ respectively with $p$ value of $<0.001$ that was statistically very significant. $1 \mathrm{~min}$ APGAR score and $5 \mathrm{~min}$ APGAR score of $<5$ had $22.2 \%$ and $11.1 \%$ contribution in neonatal mortality with $p$ value of 0.156 and 0.714 respectively.

Our study showed that neonatal mortality was not significantly affected by low APGAR score. Basu et al found that Apgar score less than or equal to 5 at one minute, apnea, gestational age, neonatal septicaemia and shock are the factors directly responsible for neonatal mortality ${ }^{5}$. Sepsis was the major contributing factor $(77.3 \%)$ of morbidity in very low birth weight neonates in our study. HMD was the second common morbidity factor present in $66.7 \%$ of the neonates. Common morbidity factors seen in our study were sepsis, HMD, NNJ, hypoglycemia, hypocalcemia. In a similar study, conducted by Mannan et al common complications of VLBW babies noted were frequent apnea (40\%), Septicemia (25.71\%), Hypothermia (17.14\%), NEC (14.28\%), Convulsion (11.43\%), Hyperbilirubinaemia (8.57\%), Anemia (5.71\%), IVH (5.71\%), RDS $(2.86 \%)$ either alone or in combination with other clinical conditions ${ }^{8}$.

The mortality among VLBW neonates in NICU, in our study was $12 \%$. Khan et al found mortality of $14 \%$ in their NICU ${ }^{10}$. In our study, many factors contribute towards neonatal mortalities. Sepsis along with other factors play an important role in neonatal mortality $(p=0.083)$. In a study done by Haque et al, it was concluded that mortality from neonatal sepsis in VLBW infants had remained between $18-20 \%$ in the developed world and around $80 \%$ in the developing world for last three decades with little sign of decline. In a study conducted by Boo, respiratory distress, intraventricular haemorrhage and sepsis were the commonest cause of death ${ }^{7}$. Hence, the common causes of death in our study are in concordance with other studies conducted worldwide.

\section{Conclusion}

The most common cause of VLBW babies was prematurity. Among maternal causes, Oligohydramnios and $\mathrm{PIH}$ were the main predisposing factors for VLBW babies. Sepsis, HMD and NNJ were the major morbidities seen among VLBW neonates. Sepsis, HMD metabolic complications like hypoglycaemia and hypocalcemia were the major contributing factors towards mortality. Prematurity was a significant cause for post natal complications like sepsis, HMD, NNJ and NEC but didn't increase risk of mortality. APGAR score $<5$ at $1 \mathrm{~min}$ and at 5 min was a significant predictor of CNS morbidities (HIE, IVH and PNA ) and overall mortality. Antenatal complications like oligohydraminos, PROM, anemia had poor neonatal outcome in form of increased risk of sepsis, PNA and mortality. Ensuring adequate antenatal care can decrease the incidence of maternal complications and thus VLBW. Appropriate methods adopted to decrease prematurity can lead to significant decline in rate of VLBW births.

\section{Acknowledgements: None \\ Funding: Nil \\ Conflict of Interest: None \\ Permission from IRB: Yes}

\section{References}

1. Bhutta ZA. Priorities in newborn care and development of clinical neonatology in Pakistan:Where to now? J Coll Physicians Surg Pak 1997;7:231-34.

2. Stewart AL,Roth SC. Neurodevelopmental Outcome. In: Rennie JM, Roberton NRC editors. Textbook of Neonatology. Edinburgh: Churchill Livingstone ;1999.p.79-100.

3. Koops B, Morgan LJ, Battaglia FC. Neonatal mortality risk in relation to birth weight and gestational age. J Pediatr 1982;101:969-77.

4. Basu S, Rathore P, Bhatia BD. Predictors of mortality in very low birth weight neonates in India. Singapore Med J 2008;49(7):556-60.

5. Lee K, Gartner LM, Eidelman Al, Ezhuthachan S. Unconjugated hyperbilirubinemia in very low birth weight infants. Clin Perinatol 1977;4(2):305-20.

6. Stoll BJ, Hansen NE, Fanaroff AA, Wright LL, Carlo WA, Ehrenkran RA, Lemons JA, Donovan EF, Stark AR, Tyson JE, Bauer CR, Shankaran S, Laptook AR, Stevenson DK, Poole WK. Change in pathogens causing early onset sepsis in VLBW infants. $N$ Engl J Med 2002;347(4):240-7.

7. Boo NY.Outcome of very low birthweight neonates in a developing country: experience from a large Malaysian maternity hospital. Singapore Med J 1992;33(1):33-7. 
8. Mannan MA, Jahan N,Dey SK, Uddin MF, Ahmed S.Maternal and foetal risk factor and complication with immediate outcome during hospital stay of very low birth weight babies. Mymensingh Med J 2012;21(4):639-47.

9. Brothwood M, Wolke D, Gamsu H, Benson J, Cooper D. Prognosis of the very low birthweight baby in relation to gender. Arch Dis Child 1986;61(6):55964.
10. Khan MR, Maheshwari PK, Shamim H, Ahmed S, Ali SR. Morbidity pattern of sick hospitalized preterm infants in Karachi, Pakistan. J Pak Med Assoc 2012;62(4):386-8.

11. Mallory $\mathrm{MH}$. Impact of cesarean section on intermediate and late preterm births: United States, 2000-2003. Birth 2009;36(1):26-33. 